\title{
Research on forgiveness characteristics development of adolescents in China.
}

\author{
Zhou Yan-Gen $^{1,2}$, Lu Jia-Mei ${ }^{{ }^{*}}$, Sang Qing-song ${ }^{3 *}$, Zhang Peng-cheng ${ }^{1}$, Wang Hai-Bin ${ }^{1}$ \\ ${ }^{1}$ Education College, Shanghai Normal University, Shanghai, PR China \\ ${ }^{2}$ Nanjing Normal University Taizhou College, Taizhou, Jiangsu, PR China \\ ${ }^{3}$ College of Education Science, Anhui Normal University, Wuhu, Anhui, PR China
}

\begin{abstract}
Objective: To provide effective basis for the forgiveness development of adolescents through the large scale investigation on the characteristics for the first time.

Methods: 2324 cases of adolescents in China were selected for the forgiveness questionnaire-Forgiveness Questionnaire of Adolescents.

Results: The results showed that the total forgiveness score of adolescents was 3.79, which was between "minor no" and "minor yes" in the positive range. Adolescents' forgiveness showed differences in learning period and gender.

Conclusion: Generally, China's adolescents' forgiveness is positive, but it needs to be improved. The total forgiveness score increases with the learning period. In-depth forgiveness increases with the learning period while surface forgiveness decreases with the learning period. The total forgiveness of male is significantly higher than that of female after middle school. The in-depth forgiveness of male is significantly lower than that of female, while surface forgiveness shows the contrary.
\end{abstract}

Keywords: Forgiveness, In-depth forgiveness, Surface forgiveness, Development characteristics.

Accepted on May 22, 2017

\section{Introduction}

Forgiveness is one of the hot topics in the psychology study. Forgiveness can help repair interpersonal relationships, promote pro-social motives, enhance social skills, and bring individuals positive psychological, emotional, and social results [1]. Research on forgiveness has attracted wide attention in academia and child psychology, with fruitful results [2]. However, China's psychological study on forgiveness is still in the initial stage. Since the definition and measurement tools of forgiveness are mostly made in the context of Western culture, the research lacks locality and positivism in China. The empirical research on adolescents' forgiveness is especially insufficient. To learn more about the characteristics of the psychological development of adolescents in China, we conducted a semi-structured interview on 368 adolescents, covering two issues. First: what do you think is forgiveness? Please define it. Second: why do you forgive others? Interview results showed that: China's adolescents generally believed that forgiveness is a positive psychological quality, which requires thinking in others place, forgiving others for damages they caused, and eliminating negative emotions. Some adolescents shared the opinion that it was difficult to forgive others and forgiveness only happened when they are requested by teachers, friends, parents, and social codes. Another section of adolescents had a view that people should be open-minded to take the initiative to forgive others.
They believed that forgiving others was like forgiving themselves. Based on the interview, we believed that forgiveness is an emotion when individuals can forgive others and got peace at the same time. Forgiveness includes in-depth forgiveness and surface forgiveness. In-depth forgiveness means the individual can take the initiative to forgive others and get peace at the same time. Whereas, surface forgiveness means that individuals can forgive others under the requests (of teachers, parents, and friends) and get peace.

In daily life, adolescents can hurt others or get hurt by others more or less, which can be physical or mental, including repeated criticism, ridicule, insult, or bully. In certain cases, people handle it in a better way to understand and tolerate others with forgiveness. In this way, they can experience happiness. On the contrary, some adolescents tend to blame others and held the grudge with negative emotions including anger, hostility, and pain. In this case, the conflict will be escalated, which might trigger vicious violence and cause negative influences on social stability and campus harmony. Therefore, in order to develop forgiveness and promote healthy growth of adolescents, it is of significant reference value to understand their development characteristics. 


\section{Research Methods}

\section{Research object}

2324 cases of adolescents in 11 schools of Nanjing, Suzhou, and Taizhou were randomly selected as objects. There were 1087 males and 1237 females; 208 in $5^{\text {th }}$ grade and 205 in $6^{\text {th }}$ grade in primary school; 210 in $7^{\text {th }}$ grade, 210 in 8 th grade, and 112 in $9^{\text {th }}$ grade of middle school; 192 in grade 1, 317 in grade 2, and 161 in grade 3 of senior middle school; 204 were freshmen, 164 were sophomores, 167 were juniors, and 174 were seniors.

\section{Research tools}

In this research, a self-compiled Adolescent Forgiveness Questionnaire was used. It is made based on the definition and constitution of forgiveness, with combining literature review and open survey through theory deduction and experience summary. The questionnaire included 14 questions and 2 factors. There were 6 levels: from 1 point for "completely no" to 6 points for "completely yes". In combination with gravel figure and characteristic value, questions with load less than 0.4 were eliminated after exploratory factor analysis. The final questionnaire included 8 questions and two factors including surface forgiveness and in-depth forgiveness. The eigenvalue of both factors were greater than 1, with explaining $51.428 \%$ of the total variation. The internal consistency coefficients of total questionnaire and both factors were $0.802,0.727$, and 0.750 . The indicators of the confirmatory factor analysis are shown in Table 1, indicating good structure of the questionnaire.

Table 1. Fit indicators of confirmatory factors analysis of adolescent forgiveness questionnaire.

\begin{tabular}{llllllllll}
\hline $\mathrm{X}^{2}$ & df & $\mathrm{X}^{2} / \mathrm{df}$ & $\mathrm{RMSEA}$ & $\mathrm{CFI}$ & $\mathrm{GFI}$ & $\mathrm{IFI}$ & $\mathrm{NFI}$ & $\mathrm{NNFI}$ & AGFI \\
\hline 54.89 & 19 & 2.89 & 0.096 & 0.94 & 0.95 & 0.94 & 0.93 & 0.91 & 0.91
\end{tabular}

\section{Data processing}

To ensure that the investigation process was strict and standard, group members and graduate students were trained and assigned to conduct field investigation rather than mandatory investigation. Divided into different classes, the objects were given unified guidance. The questionnaires were answered anonymously and handed back on site. All investigation data were entered in the computer for analysis and processing by SPSS19.0.

\section{Results}

\section{General characteristics of adolescents' forgiveness development}

The results showed that: the total forgiveness score was 3.79, surface forgives score was 4.01, and in-depth score was 3.57 (Refer to Table 2. The scores were slightly higher than the theoretical value, between "minor yes" and "minor no" within the positive range (The dividing line of positive and negative range was 3.50)).

\section{Differences in learning period and gender of adolescents' forgiveness development}

We conducted 4 (learning period) $\times 2$ (gender) multivariate analysis of variance, with total forgiveness score and two factors and dependent variables. The result showed that the main effects of learning period and gender of total forgiveness score and subordinate factors were significant $(\mathrm{P}<0.05, \mathrm{P}<0.01$, or $\mathrm{P}<0.001)$. The interaction effects of gender and grade between total forgiveness score and surface forgiveness were significant $(\mathrm{P}<0.05)$. However, the interaction effects of gender and grade of surface forgiveness were not significant $(\mathrm{P}>0.05)$. The main effect of learning period meant that the total forgiveness score and in-depth forgiveness increased with the learning period, with significant differences among various learning periods $(\mathrm{P}<0.05$ or $\mathrm{P}<0.01)$. The surface forgiveness score reduced with the learning period. The differences among various learning periods were significant besides high school and college $(\mathrm{P}<0.05$ or $\mathrm{P}<0.01)$. The main effect of gender was showed in the way that the total forgiveness score and surface forgiveness score of the male adolescents were significantly higher than female adolescents $(\mathrm{P}<0.001)$. But the in-depth forgiveness score of male adolescents was significantly lower than female adolescents $(\mathrm{P}<0.001)$. The interactive effect of the total forgiveness score and gender and grade of surface forgiveness was showed in the way that the total forgiveness score of male adolescents was significantly higher than female adolescents after middle school $(\mathrm{P}<0.05)$. But the surface forgives score of male adolescents was significantly higher than female adolescents in every learning period $(\mathrm{P}<0.05)$.

Table 2. Analysis of learning period and gender interaction of adolescents' forgiveness and subordinate factors.

\begin{tabular}{|c|c|c|c|c|c|c|}
\hline \multicolumn{2}{|c|}{ Dependent variable } & \multirow{2}{*}{$\begin{array}{l}\text { Type III sum of squares } \\
85.722\end{array}$} & \multirow{2}{*}{$\begin{array}{l}\mathbf{d f} \\
3\end{array}$} & \multirow{2}{*}{$\begin{array}{l}\text { Mean square } \\
28.574\end{array}$} & \multirow{2}{*}{$\begin{array}{l}\mathbf{F} \\
31.338\end{array}$} & \multirow{2}{*}{$\begin{array}{l}\mathbf{P} \\
0.000\end{array}$} \\
\hline Learning period & Surface forgiveness & & & & & \\
\hline & In-depth forgiveness & 462.207 & 3 & 154.069 & 178.408 & 0.000 \\
\hline & Total forgiveness score & 37.643 & 3 & 12.548 & 23.545 & 0.000 \\
\hline \multirow[t]{2}{*}{ Gender } & Surface forgiveness & 76.321 & 1 & 76.321 & 83.703 & 0.000 \\
\hline & In-depth forgiveness & 26.662 & 1 & 26.662 & 30.874 & 0.000 \\
\hline
\end{tabular}




\begin{tabular}{|c|c|c|c|c|c|c|c|}
\hline & & Total forgiveness score & 3.191 & 1 & 3.191 & 5.988 & 0.003 \\
\hline \multirow{3}{*}{$\begin{array}{l}\text { Learning } \\
\text { Gender }\end{array}$} & Period & $\times$ Surface Forgiveness & 7.791 & 3 & 2.597 & 2.848 & 0.025 \\
\hline & & In-depth forgiveness & 4.664 & 3 & 1.555 & 1.800 & 0.067 \\
\hline & & Total forgiveness score & 5.310 & 3 & 1.770 & 3.321 & 0.006 \\
\hline
\end{tabular}

\section{Analysis and Discussion}

\section{General characteristics of adolescents' forgiveness}

Based on the research results, the total forgiveness score was 3.79, which was slightly higher than the theoretical value, between "minor yes" and "minor no" within the positive range. In other words, the general adolescents' forgiveness was positive. This might be because adolescents were influenced by Chinese traditional culture that honored "forgiveness" and "being lenient wherever it is possible" along their growth. Another reason might be that Chinese social education and school education both paid attention on forgiveness. The forgive awareness of adolescents were strengthened through various methods so that adolescents learnt why and how to forgiveness others. Nevertheless, the result also showed that the forgiveness level of adolescent needs to be improved. The scores were between 3 and 4, which were not "minor yes".

\section{Significant learning period difference existed in adolescent forgiveness}

One of the major finding of the study was that the total adolescents' forgiveness score increased along with the learning period. This evidently shows that the learning period can increase the social experience of individuals. This enables them to think from others' point of view and tried to understand the importance of forgiveness. As they get exposed to more and more education materials that honored "tolerance makes peace" and "being lenient wherever it is possible" their understanding gets dramatically improved. In this case, they gradually understood the way to forgive others. Although there was no systematic research on the emotion quality of students in each learning period, the research on adolescents had shown that the emotion quality gets increased along with the learning period [3]. Thus the study implicates that increasing of forgiveness may be related to the increasing of emotion quality.

The authors also conclude that the in-depth forgiveness of adolescents increased along with the learning period. The authors thought there were several reasons for this: First, the empathy ability was enhanced. With the increasing of learning period, adolescents became more mature and be able to think for others. Thus their forgiveness ability was improved. This was consistent with the result of Zechmeister [4] which showed empathy contributed to the promotion of forgiveness through narrative studies. Second, the social cognitive ability was improved. With the increasing of learning period and age, adolescents learnt that anybody could make mistakes. They would learn in-depth forgiveness to better adapt to the society and get on well with others. As was found by Friesen, positive social cognition had a significant predictive effect on forgiveness [5].

Supporting the second finding there is a sharp decrease in surface forgiveness along with the increasing of learning period. This might be caused by the following two reasons: First, their independent consciousness was enhanced. With the increasing of learning period, adolescents were more independent. In this way, they would handle things with the way they recognized when they were hurt, rather than forgiving others just because they were asked and persuaded. Second, they were rebellious in their puberty. Puberty was characterized by more and clearer individual consciousness, desire for freedom, and rebellious psychology. In this way, adolescents seldom apologize when offended by others, which would affect their re-awareness and re-evaluation for the damage [6]. In this way, they would not be clear about the intention, which deepened their pressure and prevented surface forgiveness.

\section{Significant gender difference existed in adolescent forgiveness}

The research findings suggest that the forgiveness level of male students were significantly higher than that of female students after middle school. The nature of the conflict in both the genders was different. Studies had shown that, the nature of violation directly affected the willingness and level of forgiveness. Generally, things that hurt female are unavoidable trivial things in daily life, which made female more easily to be kept in the mood of hurt. In this way, they were more difficult to forgive that male. Second, they were influenced by adverse adaption. As was found by Charlotte et al., female in puberty who got lower scores in humanity dimension were more likely to experience adverse adaption. Agreeableness had significantly negative correlation with the bad adaption. Therefore, adverse adaption had important influence on forgiveness. In addition, this research also suggested that middle school was the critical period for male and female in terms of forgiveness, which was worthy our attention.

The results showed that the in-depth forgiveness score of male was significantly lower than that of female. The reason might lie in the following factors:

Personality: Personality had an important influence on forgiveness. In general, the personality agreeableness of male was better. However, despite the surface forgiveness, they would be "re-stimulated" because the source factor was not completely resolved. On the other hand, female featured delicate emotions. They were more likely to forgive others from the bottom of their heart. 
Empathy: Most of domestic and foreign studies showed that the empathy ability, especially emotional empathy ability of female was higher than that of male. Therefore, female were more likely to think in the perspective of others and decide to forgive. In this way, they would show in-depth forgiveness.

Attribution style: The attribution of negative behavior of the perpetrator would directly affect whether the victim should condone the injurer. People with high empathy ability tended to self-attribution. Therefore, female were more likely to reach indepth forgiveness than male.

Crisis vulnerability of psychological crisis: Crisis vulnerability is an important factor to describe and estimate the ability of individuals from negative aspect under great pressure. Compared with male, female showed lower scores in crisis vulnerability dimension. They had better psychological level in face of frustrations. This might be the reason why female showed higher scores than male.

The result of this research also showed that the surface forgiveness of male was significantly higher than that of female.

Gender roles: Gender roles were different. The social expectations for male and female were different. Male was regarded as forgivable and generous, while female enjoyed easier requirement. Therefore, male was likely to reach surface forgiveness from others' evaluation or self-evaluation.

Response: The response was different. Response was an important factor for forgiveness. Response referred to the efforts taken by individuals in cognition or behavior to get mental balance in the event of stress. In the event of conflicts and problems, female tended to choose vent and patient ways with avoiding motives. However, this couldn't solve the source of the conflict or problem. On the contrary, male was likely to use fantasy method and alleviate the conflict or problem in the fantasy. In this way, they were more likely to reach surface forgiveness.

\section{Conclusion}

To understand the development characteristics of adolescents' forgiveness, this research used Adolescents' Forgiveness Questionnaire to conduct a large scale investigation. The research found that the total forgiveness of China's adolescents was positive, with requiring improvement. The total forgiveness score increases with the learning period. The indepth forgiveness increases along with learning period, while surface forgiveness decreases along with learning period. The total forgiveness of male is significantly higher than that of female after middle school. The in-depth forgiveness of male is significantly lower than that of female, while surface forgiveness shows the contrary. Limited by time and energy, this research neglected the area of objects in the sampling, which made it hanging for further discussion to promote the research result. In the future, we will conduct a more extensive and representative sampling in China and try to establish the norm of adolescents' forgiveness, which will provide a basis for the investigation and understanding of the forgiveness development level of adolescents in China.

\section{Acknowledgment}

The work presented in this paper is supported by National social science fund Education Youth project (CEA130144): The Development Characteristics and Physiological and psychological mechanism of Chinese Teenagers' Forgiveness

\section{References}

1. Karremans JC, Van Lange PA, Holland RW. Forgiveness and its associations with prosocial thinking, feeling, and doing beyond the relationship with the offender. Personal Soc Psychol Bull 2005; 31: 1315-1326.

2. Milo M. To forgive is to be sane and realistic: contributions of REBT to the psychology of forgiving. J Rat-Emo Cognitive-Behav Ther 2015; 33: 325-340.

3. Lu JM, Liu W, He W, Yuan J, Zhu PL. An investigation of the Status quo of Chinas Contemporary Youths Affective Quality. Acta Psychologica Sinica 2016; 12: 1152-1164.

4. Zechmeister JS, Romero C. Victim and offender accounts of interpersonal conflict: Autobiographical narratives of forgiveness and unforgiveness. J Personal Soc Psychol 2002; 82: 675-686

5. Friesen MD, Fletcher GJO, Overall NC. A dyadic assessment of forgiveness in intimate relationships. Personal Relationships 2005; 12: 61-78.

6. Jeannes Z. Dont apologizes unless you mean a laboratory investigation of forgiveness and retaliation. J Soc Clin Psychol 2004; 23: 532-564.

\section{*Correspondence to}

Lu Jia-Mei

Education College

Shanghai Normal University

PR China

Sang Qing-song

College of Education Science

Anhui Normal University

PR China 